\title{
Effect of Age and Parity on Practice of Cervical Cancer Screening Among Women of Child Bearing Age in Anambra State
}

\section{Nwankwo Nonyelum Stella*}

*Correspondence: Nwankwo Nonyelum Stella

Address: Department of Human Kinetics and Health Education, Nwafor Orizu College of Education, Nsugbe Anambra State, Nigeria

e-mail $\bowtie$ nwankwostella100@gmail.com

Received: 08 October 2021; Accepted: 13 October 2021

Copyright: (C) 2021 Stella NN. This is an open-access article distributed under the terms of the Creative Commons Attribution License, which permits unrestricted use, distribution, and reproduction in any medium, provided that the original work is properly cited.

\section{ABSTRACT}

The goal of this study was to see how age and parity affected cervical cancer screening among women of childbearing age in Anambra State. The population of the study was 1,876 women of childbearing age who registered for prenatal care in public hospitals in Anambra State during the study period. Two research questions and two research hypotheses led the investigation. A multi-stage (purposive) sampling procedure was used to choose the one hundred and eighty-five (185) participants. Cronbach alpha was used to estimate the instrument's internal consistency, which was used to determine its dependability. The Statistical Package for Social Science (SPSS) was used for data input and analysis and inferential statistics (ANCOVA) was utilized to test the null hypotheses at the 0.05 level of significance. The results showed that was no significant difference in mean cervical cancer screening practice scores of women of child bearing age of different age groups. Also there was equally no significant interaction between treatment groups and parity levels on mean cervical cancer practice scores of women of child bearing age. Important conclusions and recommendations were made based on the findings.

Keywords: Age, Parity, Cervical Cancer Screening, Women of Child Bearing Age

\section{Introduction}

Cervical cancer is an abnormal illness that affects the cervix. Cervical cancer can strike any woman at any time. Those at risk of sexually transmitted diseases, such as those with multiple sex partners, early age sexual intercourse, and the presence of other sexually transmitted viruses including Herpes simplex and Human Immunodeficiency Virus infection, have higher incidences of cervical cancer (Rahman et al., 2013; Davies-Oliveira et al., 2021). Although risk factors have a role in the development of cancer, the majority of them do not cause cancer. Some people who have a number of risk factors never get cancer, whereas others who have no known risk factors do. HPV infection, immune system 
insufficiency, herpes, smoking, age, socioeconomic factors, oral contraceptives, and exposure to diethylstilbestrol (DES) can all increase a woman's risk of developing cervical cancer. Cervical cancer is also likely to rise as a result of the culture of early marriage and early sexual intercourse (Ghosh et al., 2021).

Early detection by screening is critical in the treatment of cervical cancer, however most women in developing countries arrive with advanced illness for which there is no treatment (Owoeye and Ibrahim, 2013). Several factors have been identified as contributing to late presentations, including lack of awareness of symptoms, fatalistic attitudes, willingness to attribute neoplastic disease to supernatural causes, resulting in delays in seeking help, fear of confirmation of suspicion, and, of course, the perennial problem of low population coverage by health center services, particularly in rural areas (Friebel-Klingner et al., 2021). Cervical cancer screening is included in the annual medical and health care consultations that are advised for women in developed countries. Primary prevention, on the other hand, is the prevention of HPV-related cancers and the use of the HPV vaccination to lower the risk of cervical cancer (Gulja and Gvozdanovi, 2017).

Secondary prevention entails early disease detection, such as a visit to a gynecologist and cytological analysis at the onset of sexual activity but no later than the age of 20 , because regular checks can detect sexually transmitted infections, pre-stages, and the earliest stages of cancer when it is still curable (Nguyen et al., 2021). Cervical cancer tertiary prevention is for all women who need treatment for invasive cancer at any age. Ablative surgery, radiation, and chemotherapy are all options for tertiary preventive treatment (Onyenwenyi and Gugu, 2016). Many health care professionals are concerned about the poor implementation of cervical cancer prevention policies and guidelines, and there is a lack of knowledge about the impact of structured programs on cervical cancer prevention behaviors among women of childbearing age in Anambra State. Through health education, a paradigm change is promoted for primary, secondary, and tertiary preventative interventions for cervical cancer.

Women of childbearing age in Anambra State were chosen for the study because they are generally in the age period (18-40 years) when information may be gained through formal/informal education and other techniques. This group's lack of information and bad habits when it comes to cervical cancer screening and prevention could offer substantial obstacles to the uptake of cervical cancer screening when it is appropriate (Borrull-Guardeño et al., 2021). As a result, it's critical to address bad habits and information gaps before women reach the appropriate age for screening. As a result, in order for cervical cancer screening measures to be properly employed, women of childbearing age in Anambra State must be aware of the methods' availability, as well as have a basic understanding 
of the disease and screening procedures. These may increase screening uptake, resulting in a reduction in cervical cancer-related morbidity and mortality, particularly in the state. In view of the foregoing, the researcher wishes to investigate the impact of age and parity on cervical cancer screening among women of childbearing age in Anambra State.

\section{Theoretical Framework}

The Health Belief Model is a detailed survey that aids clinicians in disease prevention. Furthermore, the foundation of this strategy is to encourage people to participate and increase their knowledge, resulting in appropriate behavior. The first phase in taking preventive measures is to recognize the danger of cervical cancer (perceived susceptibility). Following that, the severity and lifethreatening consequences of cancer, receiving good developments from the environment and positive cancer symptoms, believing in accuracy and the benefits of preventive programs, as well as the inhibitory factors of accurate behavior that are less important than its benefits, and finally performing preventive behavior, as shown in Figure 1.
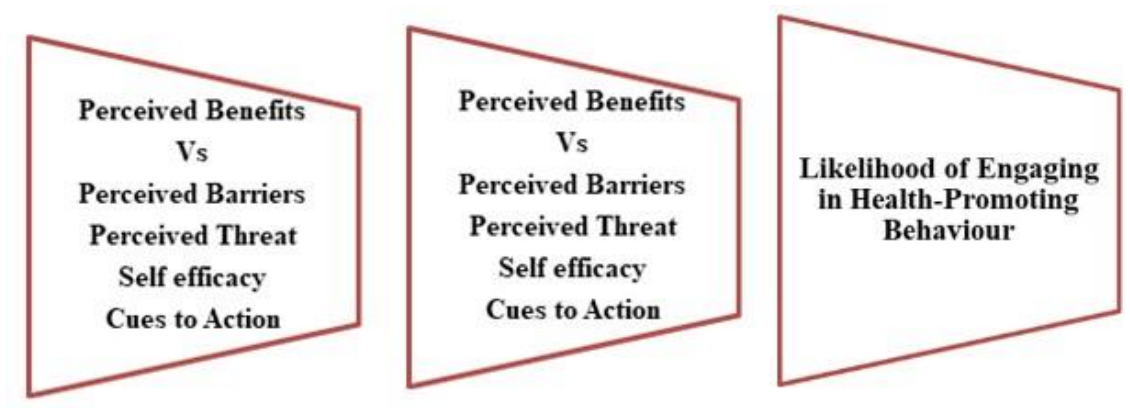

Figure 1: The Health Belief Model of Cervical Cancer Care and Management.

Estimated cervical cancer risk and net advantages, perceived cervical cancer vulnerability, rated cervical cancer intensity, supposed benefits, and perceived barriers were all covered by the HBM. These ideas were presented as a way to account for people's willingness to act, with the addition of a concept called 'cues to action,' which would trigger that willingness and promote overt behavior. The idea of 'self-efficacy,' which is a recent addition to the HBM, argues that the probability of taking action is not simply a consequence of beliefs about outcomes, but also of a person's perception that he or she is behaviorally capable of reaching the desired end (Holguin, 2009).

\section{Statement of the Problem}

Cervical cancer has a high incidence and a poor prognosis for most patients, putting a significant strain on women's health. Low screening coverage has been attributed to a number of factors, including 
limited access to and availability of screening services, screening costs, a lack of trained service providers, insufficient equipment and supplies, insufficient monitoring and evaluation of screening programs, and an overburdened health-care system. They still appear to be oblivious about the existence and probability of cervical cancer in women. The majority of women of reproductive age appear to be unaware of cervical cancer screening centers and what they entail. Despite being concerned about the health hazards associated with cervical cancer, a huge number of women appear to disregard cervical cancer screening as a precautionary measure. Inability to acknowledge the condition early appears to have resulted in mortality in many cases, which is higher than deaths from other disorders. Cervical cancer is on the rise, according to all signs, but with early diagnosis through screening and structure health promotion, the illness will not claim as several lives. Some Nigerian women tend to have cervical cancer at a later stages of the disease, where little or no treatment is available.

\section{Research Questions}

The following were the research questions that guided the study.

1. What is the pretest and posttest mean cervical cancer screening practice scores of women of child bearing age of different age groups exposed to health education programme and those not exposed?

2. What is the pretest and posttest mean cervical cancer screening practice scores of women of child bearing age of different parity exposed to health education programme and those not exposed?

\section{Hypotheses}

Specifically the following null hypothesis will guide the study.

1. There is no significant difference in the mean cervical cancer screening practice scores of women of child bearing age of different age groups exposed to health education programme and those in control group.

2. There is no significant difference in the mean cervical cancer screening practice scores of women of child bearing of different parity exposed to health education programme and those in control group.

\section{Research Design}

The research used a quasi-experimental research approach, with pre-test and post-test with a control group. The goal of the design is to figure out what causes the variables of interest to behave the way they do. Because some researchers that conducted similar studies to assess the impact of a Health 
Education program on student understanding and Attitude at Risk behavior employed quasiexperimental methodology, it was used in this study (Falade, 2010).

\section{Area of the Study}

Anambra state is the research area; Anambra is a Nigerian state in the southeast. Anambra's population is primarily made up of civil officials, businessmen, and women. Cervical cancer was ranked second (8.8\%) among the five most common cancers recorded in the state, hence the area was picked. (NAUTH Cancer Registry, 2009-2013).

\section{Population of the Study}

The study's participants were all pregnant women who registered for prenatal care in public hospitals in Anambra State during the study period. As mentioned in Appendix, Anambra State has two (2) tertiary hospitals and thirty-three (33) operational general hospitals. This group of women was chosen because they are of childbearing age and therefore vulnerable to cervical cancer. It is also expected that their way of living will have a significant impact on their health.

\section{Sample and Sampling Techniques}

The number of respondents was 184 women (134 in the experimental group and 50 in the control group) who were visiting prenatal appointments in public hospitals in Anambra State. The study employed a multi-stage method of sampling. Out of the three senatorial zones in Anambra State, a simple random sample methodology was employed to choose two senatorial zones. All public hospitals were chosen using a purposeful sampling strategy because their characteristics are similar. Following that, two public hospitals in each of the identified zones were chosen using a basic random sample technique using a fish bowl without replacement. The two hospitals chosen in each senatorial zone were assigned to the experimental and control groups using a simple random sampling approach. The prenatal days were chosen using simple random selection. The study included pregnant women who registered and attended prenatal clinics frequently in the four designated hospitals during the study period and met the inclusion criteria. The following were the inclusion criteria: the subject had to be proven pregnant and properly registered at the prenatal clinic.

\section{Instrument for Data Collection}

The cervical cancer knowledge and practice test questionnaire was used to collect data. The instrument was self-developed by the researcher after reviewing related literature. The instrument is 
divided into three parts (A, B and C). Section 'A' featured five questions about the respondents' demographic information (ie Age, level of education ethnicity parity level and occupation) Part 'B' had twenty-four questions about cervical cancer knowledge, whereas section ' $\mathrm{C}$ ' had twelve practice questions with four response options: very often, often, seldom, and never.

\section{Reliability of the Instrument}

The reliability co-efficient of the instrument was assessed by administering it to 20 pregnant women who will not be part of the study population to verify consistency of the instruments used for data collection. The women's replies on section B of the Kuder Richardson Kr.20 questionnaire were used to estimate the instrument's reliability co-efficient. The knowledge of cervical cancer, which has a co-efficient of 0.7, was analyzed using Gonbach's Alpha, which has a coefficient of 0.8. As a result, the instrument was regarded as trustworthy.

\section{Method of Data Collection}

On different days, the researcher held discussions with the six research assistants in the experimental and control hospitals about the program's procedure and timing. The pre-test was given and collected first, followed by the Health Education Program. After the program was completed, a posttest was given, and surveys were gathered. In the presence of the researcher and research assistants, illiterate respondents were instructed to complete the questionnaire by verbally answering to questions. Extraneous variables that are likely to alter the experiment's outcomes were controlled using the following measures. The researcher was in charge of training the research assistants. This was done to ensure that all trainees had the same training exposure, removing any prejudice that might occur from the trainers' personalities. To eliminate experimental bias and preserve uniformity of instruction, the researcher trained the research assistants and used a common lesson plan that was provided by the researcher. To avoid forgetting what they had learned, the effects of the pre-test on the post-test were limited by ensuring that the time for the post-test was not too long after treatment. The pre-test items were reshuffled and renumbered before being used for the post-test.

\section{Method of Data Analysis}

In the Statistical Package for Social Science, mean and standard deviation were utilized to answer the research questions, and inferential statistics (ANCOVA) was employed to test the null hypotheses at the 0.05 level of significance (SPSS). The variables were appropriately labeled and the raw data was loaded into the SPSS sheet. The research objectives were used to guide the analysis. 


\section{Results and discussions}

Research Question 1: What is the pretest and posttest mean cervical cancer screening practice scores of women of child bearing age of different age groups exposed to health education programme and those not exposed?

Table 1: Pretest and Posttest Mean Cervical Cancer Screening Practice Scores and Standard Deviation of Women of Child Bearing Age of Different Age Groups Exposed to Health Education Programme and those in Control Group.

\begin{tabular}{|c|c|c|c|c|c|c|c|}
\hline \multirow[t]{2}{*}{ Age Groups } & \multicolumn{3}{|c|}{ Pre test } & \multicolumn{3}{|c|}{ Post test } & \multirow[t]{2}{*}{ Mean Gain Score } \\
\hline & $\mathbf{N}$ & Mean & SD & $\mathbf{N}$ & Mean & SD & \\
\hline \multicolumn{8}{|l|}{ Health Education } \\
\hline \multicolumn{8}{|c|}{ Programme Group: } \\
\hline $18-28$ years & 64 & 15.81 & 7.09 & 64 & 19.33 & 6.90 & 3.51 \\
\hline 29-39 years & 67 & 16.76 & 7.59 & 67 & 19.25 & 8.28 & 2.49 \\
\hline $40-50$ years & 3 & 17.67 & 9.87 & 3 & 24.67 & 7.23 & 7.00 \\
\hline \multicolumn{8}{|l|}{ Control Group: } \\
\hline $18-28$ years & 22 & 15.50 & 10.46 & 22 & 13.64 & 10.15 & -1.86 \\
\hline 29-39 years & 22 & 15.86 & 10.53 & 22 & 16.45 & 8.85 & -0.59 \\
\hline $40-50$ years & 6 & 13.83 & 9.56 & 6 & 8.17 & 7.65 & -5.66 \\
\hline \multicolumn{8}{|l|}{ Total: } \\
\hline $18-28$ years & 86 & 15.73 & 8.02 & 86 & 17.87 & 8.18 & 2.14 \\
\hline 29-39 years & 89 & 16.54 & 8.36 & 89 & 18.56 & 8.46 & 2.02 \\
\hline $40-50$ years & 9 & 15.11 & 9.23 & 9 & 13.67 & 10.85 & -1.44 \\
\hline
\end{tabular}

The results in Table 1 shows pretest, posttest mean cervical cancer screening knowledge and mean gain scores of child bearing age based on their treatment groups and age groups. Mean cervical cancer practice score based on age groups shows that the pretest and posttest mean cervical cancer screening practice scores of those within ages of 18 - 28 years were 15.73 and 17.87 with a mean gain score of 2.14. Those within the age group of 29-39 years had mean scores 16.54 and 18.56 as pretest and posttest respectively and a mean gain score of 2.02 while those within 40 and 50 years had pretest and posttest mean knowledge scores of 15.11 and 13.67 and a mean loss of -1.44 . This suggests that women within the youngest category (18-28 years) had slightly higher gain score than others.

In terms of treatment groups, women of child bearing of different age groups exposed to health education programme had higher mean cervical cancer screening practices than those of similar age groups in control group. This was demonstrated by the mean gain scores of 3.51, 2.49 and 7.00 for women between the ages of 18-28 years, 29-30 years and 40-50 years respectively. However, women within the ages of 40-50 years had the highest mean gain in cervical cancer practice score, suggesting that while the health education programme was effective for women of age groups, those within the oldest age group benefitted most. 
The funding of this study revealed that women of child bearing age of different age groups in the treatment groups exposed to health education programme had higher mean gain of 3.51 (18-28years) 2.49 (29-39years) and 7.00 (40-50 years) cervical cancer screening practices than those of similar age groups in control groups. The result was similar to the findings of Santos, Sa'couto and Hespahnol (2017) where the results showed that women within the ages of 40-50 years had the highest mean gain in cervical cancer practice score, suggesting that while the health education programme was effective for women of all age groups, those within the oldest age group benefitted most. Kitano and Yamamoto (2020) suggest that experience play a significant role in learning in health knowledge assimilation.

Research Question 2: What is the pretest and posttest mean cervical cancer screening practice scores of women of child bearing age of different parity exposed to health education programme and those not exposed?

Table 2: Pretest and Posttest Mean Cervical Cancer Screening Practice Scores and Standard Deviation of Women of Child Bearing Age of Different Parity Exposed to Health Education Programme and those in Control Group.

\begin{tabular}{|c|c|c|c|c|c|c|c|}
\hline \multicolumn{4}{|c|}{ Pre test } & \multicolumn{3}{|c|}{ Post test } & \multirow[t]{2}{*}{ Mean Gain Score } \\
\hline Parity Level & $\mathbf{N}$ & Mean & SD & $\mathbf{N}$ & Mean & SD & \\
\hline \multicolumn{8}{|c|}{ Health Education } \\
\hline \multicolumn{8}{|c|}{ Programme Group: } \\
\hline No Child & 3 & 20 & 4.36 & 3 & 22.67 & 2.52 & 2.67 \\
\hline 01-Mar & 75 & 17.13 & 7.9 & 75 & 20.13 & 7.62 & 3 \\
\hline 04-Jun & 22 & 13.36 & 5.27 & 22 & 18.68 & 7.7 & 5.32 \\
\hline 07-Sep & 7 & 15.29 & 9.05 & 7 & 14.43 & 7.28 & -0.86 \\
\hline $10 \&$ above & 27 & 16.37 & 6.72 & 27 & 18.93 & 7.79 & 2.56 \\
\hline \multicolumn{8}{|c|}{ Control Group: } \\
\hline No Child & 7 & 13.71 & 11.4 & 7 & 11.71 & 10.29 & -2 \\
\hline 01-Mar & 22 & 13.32 & 9.92 & 22 & 16.14 & 9.95 & 2.82 \\
\hline 04-Jun & 14 & 18.5 & 8.09 & 14 & 13.79 & 7.64 & -4.71 \\
\hline 07-Sep & 5 & 12.4 & 11.78 & 5 & 6.8 & 9.34 & -5.6 \\
\hline $10 \&$ above & 2 & 31.5 & 6.36 & 2 & 23.5 & 6.36 & -8 \\
\hline \multicolumn{8}{|l|}{ Total: } \\
\hline No Child & 10 & 15.6 & 10 & 10 & 15 & 10 & -0.6 \\
\hline 01-Mar & 97 & 16.27 & 8.5 & 97 & 19.23 & 8.32 & 2.96 \\
\hline 04-Jun & 36 & 15.36 & 6.89 & 36 & 16.78 & 7.94 & 1.42 \\
\hline 07-Sep & 12 & 14.08 & 9.87 & 12 & 11.25 & 8.72 & -2.83 \\
\hline $10 \&$ above & 29 & 17.41 & 7.66 & 29 & 19.24 & 7.69 & 1.83 \\
\hline
\end{tabular}

In terms of treatment groups and parity, although all parity levels parity levels exposed to health education programme had mean gain score ranging from 2.56 - 5.32, only women with 7- 9 children had mean loss in the practice of cervical cancer screening. These suggest that women of all parity levels 
benefitted from the health education programme except those with 7-9 children whereas those with 4 6 children benefitted most from the health education programme (Table 2).

Nevertheless, women with 1-3 children improved more in cervical cancer practice than women in other categories. Makuza, et al. (2015) found low prevalence and risk factors for cervical cancer and pre-cancer ons lesions among low party Rewanda women between 30 and 50 years old undergoing screening. Henke, et al. (2021) recorded that women who have had 3 or more full term pregnancies have an increased risk of developing cervical cancer. These might be due to the increase exposure to human papilloma virus infection (HPV) with sexual actuality.

Hypothesis 3: There is no significant difference in the mean cervical cancer screening practice scores of women of child bearing age of different age groups exposed to health education programme and those in control group.

Table 3: Summary of Analysis of Covariance of Child Bearing Mothers' Mean Cervical Cancer Practice Scores by Treatment Groups and Age Groups.

\begin{tabular}{|c|c|c|c|c|c|c|}
\hline Source of variation & Type III Sum of Squares & df & Mean Square & $\mathbf{F}$ & P-value & Decision \\
\hline Corrected Model & 3122.748 & 6 & 520.458 & 9.207 & 0 & \\
\hline Intercept & 3166.299 & 1 & 3166.299 & 56.014 & 0 & \\
\hline Pre-Practice Scores & 1719.589 & 1 & 1719.589 & 30.421 & 0 & S \\
\hline Groups & 855.867 & 1 & 855.867 & 15.141 & 0 & S \\
\hline Age & 45.059 & 2 & 22.529 & 0.399 & 0.672 & NS \\
\hline Groups * Age & 309.886 & 2 & 154.943 & 2.741 & 0.067 & NS \\
\hline Error & 10005.25 & 177 & 56.527 & & & \\
\hline Total & 72744 & 184 & & & & \\
\hline Corrected Total & 13128 & 183 & & & & \\
\hline
\end{tabular}

The results presented in Table 3 shows that there was no significant difference in mean cervical cancer screening practice scores of women of child bearing age of different age groups, $F(2,177)=.399$, $\mathrm{P}>0.05$. Hence, the null hypothesis was not rejected. There was equally no significant interaction between treatment groups and age on the women's cervical cancer practice, $F(2,177)=2.741, P>0.05$. The plots of means of cervical cancer screening practice scores of women of child bearing by treatment groups and different age groups is displayed in Figure 2 shows no interaction effect.

Hypothesis 4: There is no significant difference in the mean cervical cancer screening practice scores of women of child bearing of different parity exposed to health education programme and those in control group. 


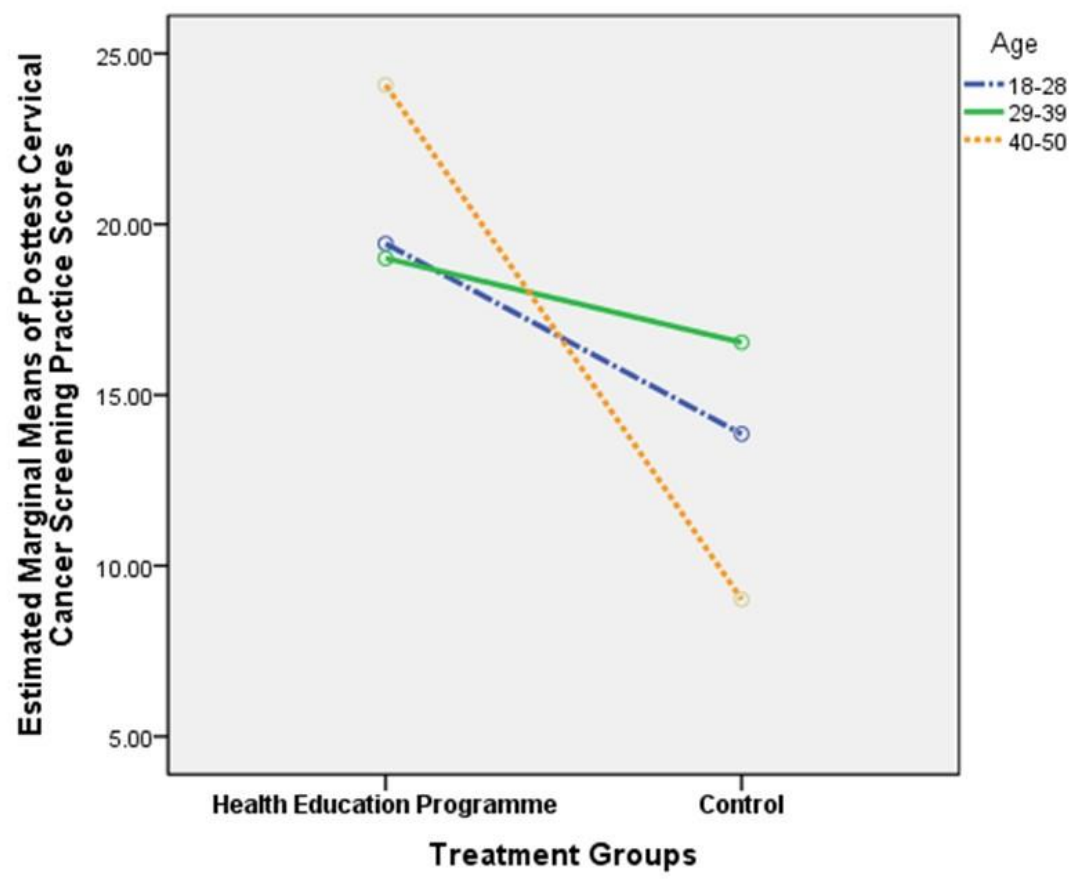

Figure 2: Mean plots of cervical cancer screening practice scores of women of child bearing age by treatment groups and age groups showing no significant interaction effect.

Table 4: Summary of Analysis of Covariance of Child Bearing Mothers' Mean Cervical Cancer Practice Scores by Treatment Groups and Parity Levels.

\begin{tabular}{|c|c|c|c|c|c|c|}
\hline $\begin{array}{l}\text { Source of } \\
\text { Variation }\end{array}$ & Type III Sum of Squares & df & Mean Square & $\mathbf{F}$ & P-value & Decision \\
\hline Corrected Model & 3387.042 & 10 & 338.704 & 6.015 & 0 & \\
\hline Intercept & 2528.057 & 1 & 2528.057 & 44.898 & 0 & \\
\hline Pre-Practice Scores & 1568.391 & 1 & 1568.391 & 27.855 & 0 & \\
\hline Groups & 427.336 & 1 & 427.336 & 7.59 & 0.006 & $\mathrm{~S}$ \\
\hline Parity Level & 528.225 & 4 & 132.056 & 2.345 & 0.057 & NS \\
\hline $\begin{array}{l}\text { Groups * Parity } \\
\text { Level }\end{array}$ & 177.642 & 4 & 44.41 & 0.789 & 0.534 & NS \\
\hline Error & 9740.958 & 173 & 56.306 & & & \\
\hline Total & 72744 & 184 & & & & \\
\hline Corrected Total & 13128 & 183 & & & & \\
\hline
\end{tabular}

The results presented in Table 4 shows that there was no significant difference in mean cervical cancer screening practice scores of women of child bearing age of different parity levels, $F(4,173)=$ $.399, \mathrm{P}>0.05$. Therefore, the null hypothesis was not rejected. There was equally no significant interaction between treatment groups and parity levels on mean cervical cancer practice scores of women of child bearing age $\mathrm{F}(2,173)=.789, \mathrm{P}>0.05$. The mean plots of cervical cancer screening practice scores of women of child bearing by treatment groups and different age groups as displayed in Figure 3 shows no significant interaction effect. Although those with 10 children and above in health 
education programme group had the least increase in mean cervical cancer screening practice score, it was better than those in control group.

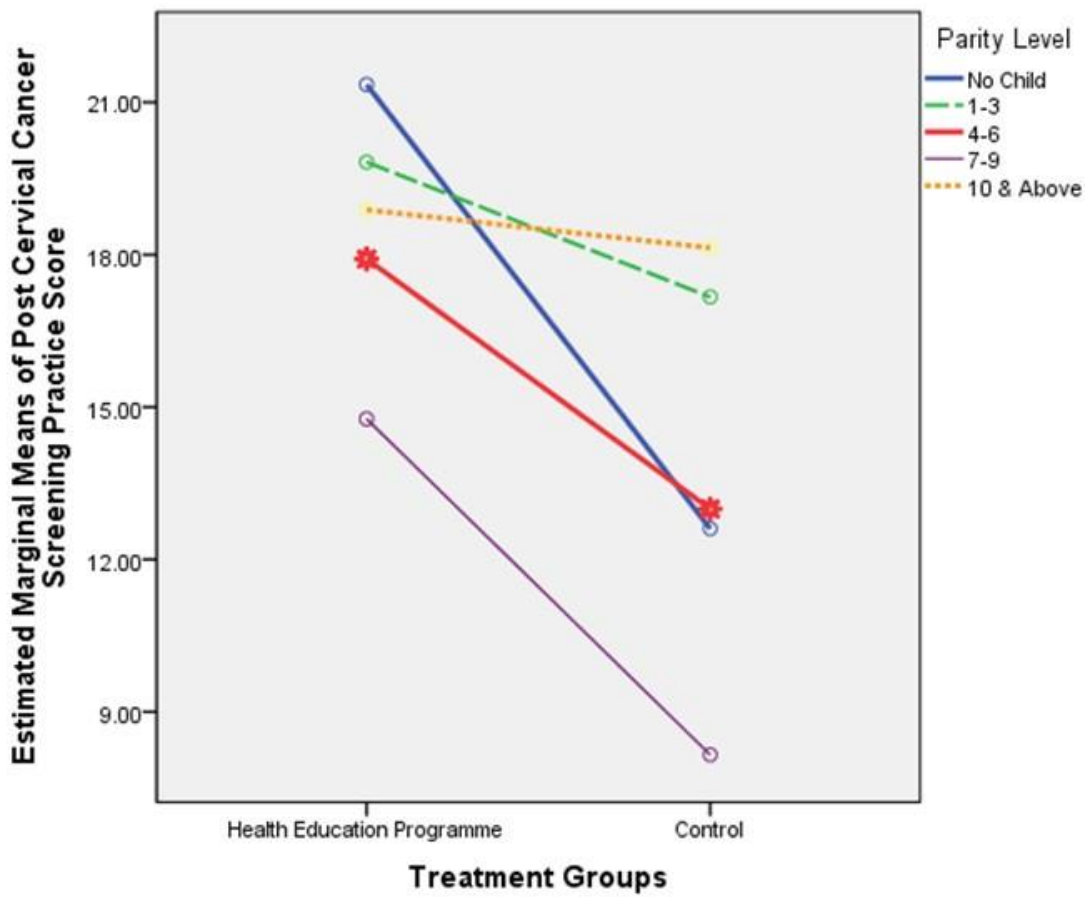

Figure 3: Mean plots of cervical cancer screening practice scores of women of child bearing age by treatment groups and parity levels showing no significant interaction effect.

\section{Implication of Findings}

This study's conclusions have a lot of educational ramifications. The findings of this study provide information on the impact of a Health Education program on women's understanding and practice of cervical cancer screening. Cervical cancer screening can help to minimize cancer's incidence and death by detecting and treating premalignant lesions, as well as promoting prompt treatment of malignant lesions with less morbidity. The outcomes of this research will be extremely beneficial to the federal, state, and local governments. This research will promote the availability of government-funded cervical cancer screening and treatment programs for the most vulnerable people. Clinical preventative care, such as cervical cancer screening and a thorough health education campaign, should be covered. Government-provided power point presentations could be an efficient way to improve knowledge and practice during prenatal and postnatal visits.

Because women with the highest educational levels had more knowledge of cervical cancer screening than those with lower levels of education, health educators can plan the goal of pap smear screening of cervical cancer while taking into account the patient's educational level, improve the 
educator-patient relationship, and increase the patient's sense of well-being by developing mutually agreed screening goals. Above all, the study's findings will aid in the expansion of the scientific body of professional information on which future research on women's cervical cancer screening expertise can be undertaken.

\section{Recommendations}

1. Because men are the decision-makers in most households and offer financial support, it is critical to encourage partner participation in cervical cancer screening.

2. Organized cervical cancer screening programs should be brought closer to communities to lower the costs associated with transportation and distance for women. Health care providers should also be trained in communication skills so that they can be pleasant when dealing with consumers, such as ladies seeking cervical cancer screening.

3. Public awareness campaigns and education programs regarding cervical cancer screening should cover a wide range of topics, including signs and symptoms, risk factors, and ways to avoid the illness.

4. Future research might look into novel ways of conveying information, such as health talks for young women and men in schools and colleges, market outreach, and health briefs at health facilities while women wait to see a doctor.

5. The Ministry of Health should collaborate with schools, churches, higher education institutions, and the general public to increase screening uptake.

\section{Conclusions}

Women within the ages of 40-50 years had the highest mean gain in cervical cancer practice score, suggesting that while the health education programme was effective for women of age groups, those within the oldest age group benefitted most. Also there was no significant difference in mean cervical cancer screening practice scores of women of child bearing age of different age groups. Specifically, the study showed that women of all parity levels benefitted from the health education programme except those with 7-9 children whereas those with $4-6$ children benefitted most from the health education programme. There was equally no significant interaction between treatment groups and parity levels on mean cervical cancer practice scores of women of child bearing age.

It can be inferred that the salient challenges that undermine cervical screening and early help 
seeking for cervical cancer symptoms with subsequent late diagnoses of cervical cancer in Anambra State may be associated with poor health education programmes especially among the younger women. It is also concluded that most women are knowledgeable about cervical cancer and are unable to go for screening services due to low cervical cancer screening knowledge. Public health education programs in communities may need to include accurate information about the causes, symptoms and prognosis of cervical cancer so that the negative attitudes and stigma associated with cervical cancer as well as patient abandonment may be reduced or prevented. This is because cervical cancer patients may present late in hospitals not because they had never had any contacts with health workers before but because lower level health workers did not suspect cancer and were providing other treatments, e.g. for sexually transmitted infections (STIs).

\section{References}

Borrull-Guardeño J, Sebastiá-Laguarda C, Donat-Colomer F, Sánchez-Martínez V. Women’s knowledge and attitudes towards cervical cancer prevention: A qualitative study in the Spanish context. J Clin Nurs 2021; 30: 1383-1393.

Davies-Oliveira JC, Smith MA, Grover S, Canfell K, Crosbie EJ. Eliminating cervical cancer: progress and challenges for high-income countries. Clin Oncol 2021; 33: 550-559.

Falade E. Understanding Attitudes and Predicting Social Behaviour. Englewood-Cliffs, 2010; NJ: Prentice-Hall.

Friebel-Klingner TM, Luckett R, Bazzett-Matabele L, Ralefala TB, Monare B, Nassali MN, Ramogola-Masire D, Bvochora M, Mitra N, Wiebe D, Rebbeck TR. Clinical and sociodemographic factors associated with late stage cervical cancer diagnosis in Botswana. BMC Womens Health 2021; 21: 1-9.

Ghosh S, Mallya SD, Shetty RS, Pattanshetty SM, Pandey D, Kabekkodu SP, Satyamoorthy K, Kamath VG. Knowledge, Attitude and Practices Towards Cervical Cancer and its Screening Among Women from Tribal Population: a Community-Based Study from Southern India. J Racial Ethn Health Disparities 2021; 8: 88-93.

Guljaš Slivečko I and Gvozdanović Z. Knowledge and Behaviour of Female Students in Relation to Cervical Cancer Prevention. Croat Nurs J 2017; 1:39-49.

Henke A, Kluge U, Borde T, Mchome B, Serventi F, Henke O. Tanzanian women's knowledge about Cervical Cancer and HPV and their prevalence of positive VIA cervical screening results. Data from a Prevention and Awareness Campaign in Northern Tanzania, 2017-2019. Glob Health Action 2021; 14: 1852780.

Holguin AC. Determining the knowledge and attitudes of 18- to 26-year-old women regarding cervical cancer, human Papillomavirus, and the human Papillomavirus vaccine, Master thesis, Health, Physical Education and Recreatio, Utah State University 2009; pp: 27-30.

Kitano S and Yamamoto N. The role of consumer knowledge, experience, and heterogeneity in fish consumption: Policy lessons from Japan. Journal of Retailing and Consumer Services 2020; 56: 102151.

Makuza JD, Nsanzimana S, Muhimpundu MA, Pace LE, Ntaganira J, Riedel DJ. Prevalence and risk factors for cervical cancer and pre-cancerous lesions in Rwanda. Pan Afr Med J 2015; 22(1).

Nguyen DN, Vu HM, Nguyen QN, Nguyen CT, Do HT, Vu TM, Tran BX, Latkin CA, Ho CS, Ho R. Gaps of Knowledge about HIV and Sexually Transmitted Infections Among Industrial Workers in Northern Vietnam. AIDS Behav 2021; pp:1-8. 
Onyenwenyi AO and Gugu GM. Strategies for the prevention and control of cervical cancer in rural communities: a Nigerian perspective. Journal of Community Medicine and Primary Health Care 2016; 28: 77-93.

Owoeye IO and Ibrahim IA. Knowledge and attitude towards cervical cancer screening among female students and staff in a tertiary institution in the Niger Delta. International Journal of Medicine and Biomedical Research 2013; 2: 48-56.

Rahman M, Mia AR, Haque SE, Golam M, Purabi NS, Choudhury SA (2013). Beating Cervical Cancer in the Developed Countries: A Dream or a Reality? In Current Topics in Public Health. IntechOpen 2013; 15: 341-358.

Santos P, Sá L, Couto L, Hespanhol A. Health literacy as a key for effective preventive medicine. Cogent Social Sciences 2017; 3: 1407522. 